\title{
Arbor
}

\section{La Armada y sus retos}

\section{Francisco Torrente Sánchez}

Arbor CLXXIII, 682 (Octubre 2002), 231-237 pp.

Se resumen en esta artículo los tres retos más significativos a los que tiene que hacer frente la Armada en los próximos años, como consecuencia del nuevo escenario estratégico internacional, del proceso de plena profesionalización de las Fuerzas Armadas, y de la consiguiente racionalización orgánica y estructural que todo ello demanda.

El Almirante Jefe del Estado Mayor de la Armada analiza cada una de estas tres áreas y traza las líneas generales de actuación para conseguir mantener y operar una Fuerza Naval moderna y equilibrada, cuya existencia y eficacia son la razón de ser de la Armada como componente de la necesaria acción conjunta en las misiones de Seguridad y Defensa encomendadas a las Fuerzas Armadas.

\section{Introducción}

Las decisiones tomadas desde hace años por la Armada en relación con los programas de modernización han ido cristalizando en una marina moderna, alcanzada con un gran esfuerzo y no pocos sacrificios, y han logrado situarla a un nivel operativo comparable con el de la mayoría de las marinas de nuestros socios y aliados europeos.

Tres son los retos más importantes a los que se enfrenta la Armada en el próximo futuro.

En primer lugar, el actual escenario estratégico internacional. La mayor presencia de España en el exterior y las nuevas misiones de 
mantenimiento de paz y de gestión de crisis exigen nuevas capacidades militares, cuya adquisición se prevé abordar en el ámbito conjunto desde unos nuevos criterios de planeamiento.

En segundo término, el proceso de plena profesionalización, que ha dado lugar a un profundo reajuste de las plantillas de las Fuerzas Armadas y requiere un cambio de mentalidad, además de exigir mejorar las condiciones de vida y las expectativas de futuro de nuestros profesionales.

En tercer lugar, y dentro del proceso de racionalización de la actual organización y estructura de los Ejércitos y de la Armada, en breve plazo se actualizarán las disposiciones sobre la estructura territorial de los tres Ejércitos, para modernizarla conforme a los criterios funcionales que establece la Directiva de Defensa Nacional del Presidente del Gobierno.

Pero todo este proceso de adaptación no es un caso exclusivo de España. Hoy, a la Armada, se le plantean unos retos de carácter general similares a los que se enfrentan el resto de nuestros aliados: cómo mantener y mejorar en lo posible el nivel alcanzado y cómo adquirir las capacidades navales adicionales que exigen las nuevas misiones.

\section{La Armada en el entorno estratégico}

La Armada desempeña un papel cada vez más relevante en el nuevo escenario estratégico internacional y contribuye, hoy más que nunca, al fortalecimiento de la creciente presencia de España en los foros internacionales.

La estrategia naval española se concibe como aportación a la estrategia conjunta nacional y a la estrategia aliada, inspiradas ambas en los conceptos de Seguridad Compartida y Defensa Colectiva. Junto a su visión naval, la Armada aporta a estas estrategias la experiencia acumulada a lo largo de décadas de interacción, adiestramiento y colaboración con marinas extranjeras.

Nuestra contribución al planeamiento militar tiene como eje central de actuación el convencimiento de que, en el futuro previsible, las operaciones en las que la participación de la Armada es más probable serán las de proyección de fuerza en un ámbito conjunto o multinacional. Para este tipo de operaciones es precisamente para lo que nos preparamos, desde el convencimiento de que la eficacia del conjunto es función del valor de cada uno de los elementos que lo componen. 
En este escenario, el conflicto armado limitado es el supuesto que debe tener mayor influencia en el diseño realista de nuestros Ejércitos y, por lo tanto, también en el de la Armada, con el fin de disponer de unas capacidades adecuadas para afrontar con garantía los riesgos del futuro previsible, tanto junto con nuestros socios y aliados como en el plano puramente nacional.

La zona de actuación más probable de las Fuerzas Armadas españolas, y en particular de la Armada, es la región euroatlántica, en la que se circunscribe el entorno geoestratégico natural de España, es decir, Europa, el Mediterráneo occidental y el Atlántico oriental, que incluye el Archipiélago Canario y el Estrecho de Gibraltar con sus accesos, punto focal de máximo interés para España. No obstante, estamos preparados para actuar en cualquier lugar del mundo donde el Gobierno decida emplearnos.

Las unidades deben estar preparadas para hacer frente a cualquier contingencia y ser capaces de minimizar los daños propios y los colaterales producidos por nuestras operaciones. Además, para obtener el mayor aprovechamiento, deben ser operativamente flexibles, capaces de responder indistintamente tanto a nuestras necesidades nacionales en el ámbito marítimo próximo como a las derivadas del escenario estratégico internacional, dentro de la opción multinacional elegida por España.

En el horizonte a medio plazo, es evidente que los nuevos conflictos se distinguirán por su carácter asimétrico, y para afrontarlos con éxito se precisará una gran superioridad tecnológica, por lo que la Armada enfatiza la calidad más que la cantidad de sus unidades, sin renunciar en ningún caso a un deseable equilibrio.

\section{Líneas generales de actuación}

Para mantener y operar una marina moderna y plenamente profesional son necesarios nuevos planteamientos, nuevos enfoques y nuevas soluciones. Las decisiones tendrán que ser meditadas cuidadosamente, teniendo bien presente que la Armada es hoy mucho menos autónoma que en el pasado pues está mucho más integrada en lo conjunto; que la disponibilidad de recursos humanos se irá incrementando progresivamente, aunque hoy por hoy no permita completar las plantillas de todas las unidades; que sólo se puede aspirar a un incremento moderado del recurso financiero; y por último que, como consecuencia de todo ello, sólo podemos llegar a unos planes 
apropiados, aceptables y practicables, tomando conciencia de que es necesario optimizar la gestión de los recursos y establecer prioridades muy rigurosas.

Las nuevas capacidades navales de la Armada deben constituir una meta pragmática, adecuada y sostenible; un compromiso entre riesgos, misiones previsibles, necesidades y recurso financiero disponible. Hacia esta meta han de converger todos los esfuerzos en inversión. Se debe impulsar la obtención de una Fuerza Naval que sirva al doble propósito de satisfacer las necesidades nacionales y contribuir a la estrategia aliada en sus justas proporciones. Para ello se necesita una Fuerza Naval de suficiente entidad y equilibrada en capacidades. En línea con las actuales necesidades estratégicas, se debe llegar a disponer de unas Fuerzas Navales flexibles, aptas para la acción conjunta, y adecuadamente orientadas a la participación en operaciones de proyección desde la mar sobre tierra.

Para poder llevarlas a cabo se requiere un grado de autoprotección adecuado ante eventuales amenazas, particularmente en aguas próximas a costa, y una autonomía logística suficiente para operar durante largos períodos de tiempo en teatros alejados.

Para ser un instrumento eficaz de nuestra estrategia de Seguridad Compartida y Defensa Colectiva, la Fuerza Naval tiene que ser, además, interoperable con la de nuestros aliados. Dentro de esta característica clave de interoperabilidad se incluye la necesidad de que nuestras autoridades navales estén en condiciones de ejercer el mando y control de fuerzas multinacionales y conjuntas, especialmente en los teatros de nuestro mayor interés estratégico.

La viabilidad de la Fuerza Naval, medida tradicionalmente en términos puramente económicos, está hoy también condicionada por el recurso de personal profesional. Sólo podemos aspirar a obtener aquellas unidades que podamos dotar apropiadamente, pues la eficacia operativa de nuestras unidades está directamente condicionada por la cobertura de sus plantillas con el personal adecuado en calidad y cantidad.

La eficacia de cualquier organización, y por supuesto la de la Armada, está absolutamente condicionada por el valor de su recurso humano, en todos sus niveles, categorías y empleos. Hoy, como siempre, la Armada vale lo que vale su gente. Pero si, hasta ahora, las necesidades de marinería y tropa estaban aseguradas por el reclutamiento forzoso, hoy debemos reclutar mediante un proyecto atractivo. Esto es lo realmente novedoso, y en este terreno no tenemos dividendos que recoger como resultado de decisiones anteriores, sino un reto importante en el que está en juego nuestro futuro. 


\section{EL futuro de Plena Profesionalización}

Hoy existe un déficit de marineros y soldados profesionales que influye directamente sobre la operatividad y sobre la carga de trabajo de nuestra gente. Por ello, en el momento actual, la culminación con éxito del proceso de profesionalización constituye la primera prioridad para la asignación de nuestros recursos.

Para cubrir con personal adecuado las plantillas, tanto de cuadros de mando como de marinería y tropa, es necesario presentar a los jóvenes españoles una oferta de trabajo atractiva y rigurosa, que tiene que contemplar cuatro aspectos fundamentales: perspectivas de futuro, integración, satisfacción en el trabajo y calidad de vida.

Las perspectivas de futuro deben ser tales que el ingreso en la Armada garantice posibilidades reales de alcanzar un futuro estable, ya sea en la carrera militar o reincorporándose al mercado laboral en las mejores condiciones de preparación una vez finalizado el tiempo de servicio.

Los marineros y soldados que deseen permanecer en la Armada deben tener probabilidades razonables de conseguirlo y suficientes expectativas de promoción. Los procedimientos de evaluación y clasificación deben ser objetivos y transparentes, para permitir que quienes demuestren un rendimiento satisfactorio puedan, si lo desean, acceder a una relación de servicios de carácter permanente.

Para quienes quieran reincorporase a la vida civil, es preciso conseguir que el haber pertenecido a la Armada suponga un factor de prestigio en el mundo laboral. Esto implica, en primer lugar, la necesidad de que la propia Armada proyecte una imagen pública de eficacia. También es necesario que nuestro personal retorne al mundo laboral con una formación útil para sus nuevas tareas, razón por la cual nuestros planes de estudios deben incluir materias no específicas de la Armada. Además, el modelo de carrera debe permitir alternar los destinos de embarque con otros en los que sea posible acceder a formación adicional.

El objetivo de la integración es conseguir que todos los hombres y mujeres de la Armada, incluido el personal civil, la sientan como algo suyo, una Institución de la que puedan sentirse orgullosos en la misma medida que la Armada se siente orgullosa de su gente. Ello requiere la mayor transparencia y la difusión, a todos los niveles, de todo lo que se relaciona con los objetivos y la política naval, especialmente en sus aspectos de personal.

La satisfacción en el trabajo exige una formación de calidad, auténtico capital que la Armada debe ofrecer a todos sus componentes. 
Exige, además, que las tareas asignadas a cada uno estén en consonancia con la formación recibida, y que el entorno de trabajo les permita tomar las decisiones de su competencia y se responsabilicen de su cometido.

Estas pautas de comportamiento, que no son nuevas pero que con la profesionalización cobran mayor importancia, hacen necesario definir un modelo de liderazgo participativo e integrador, que debe ser exigido a todos los profesionales, y en especial a los cuadros de mando.

En cuanto a la calidad de vida, hay que señalar que la dureza de la vida en la mar, la separación temporal del entorno familiar y la movilidad geográfica son dificultades que la Armada no puede eliminar, pero cuyas consecuencias se tratan de paliar y compensar en la medida de lo posible. Mejorar la calidad de vida, no sólo de los marineros y soldados profesionales, sino de todo el personal de la Armada, exige mayores inversiones en infraestructura y en la contratación externa de ciertas tareas, así como la racionalización de guardias y servicios, y la adaptación del régimen interior de las unidades a las exigencias de una marina moderna y totalmente profesional.

Las mayores necesidades de formación que se derivan de la plena profesionalización deben reflejarse en una mayor dotación económica a las Escuelas, en un mayor grado de cobertura de sus plantillas en cantidad y en calidad, en iniciativas para la explotación de nuevas tecnologías en la enseñanza, y en el impulso a los estudios sobre racionalización y concentración de Escuelas.

\section{Evolución orgánica y racionalización de la estructura}

El proceso de racionalización de las estructuras de las Fuerzas Armadas responde a criterios funcionales. El efecto más inmediato de este proceso será la desaparición de la Estructura Territorial que responde a criterios ya antiguos y desfasados, pues aunque el concepto de Zona Marítima responde más a criterios estratégicos y de despliegue de fuerzas que a criterios territoriales, no cabe duda de que los actuales Mandos de Zona, que tienen responsabilidades en zonas geográficas definidas y ejercen la autoridad militar sobre las dependencias ubicadas en sus respectivas Zonas, ligan de alguna manera sus competencias al territorio.

Las posibilidades de los modernos sistemas de Mando y Control permiten que las actuales Zonas Marítimas evolucionen hacia una entidad marítima única, que abarcará todo el litoral español, y hacen posible el control centralizado de todas las unidades que se dedican a misiones específicas de carácter permanente, bajo una única autoridad 
responsable de la parte que corresponde a la Armada en la Acción del Estado en la Mar.

La presencia de la Armada en el litoral se mantendrá en la medida necesaria, pero cada organismo estará encuadrado en la organización funcional que corresponda. De esta forma, el mando sobre las dependencias periféricas se ejercerá siguiendo las líneas de dependencia que encabezan los Almirantes responsables de la Fuerza y del Apoyo a la Fuerza.

\section{Recursos financieros}

La primera prioridad a la hora de la asignación de los recursos de la Armada es la culminación del proceso de plena profesionalización. Por ello, y hasta alcanzar este objetivo, las actividades relacionadas con este proceso, en particular en las áreas de formación, calidad de vida y externalización, tienen prioridad sobre otras necesidades en la asignación de recursos económicos.

En un segundo término, en un escenario estratégico como el que vivimos, en el que la utilidad de la Armada como instrumento de la Política Exterior depende en buena manera de que se mantenga nuestra superioridad tecnológica, la continuidad del proceso de modernización, en busca de ese Objetivo de Fuerza realista y alcanzable al que aspiramos, es también prioritaria.

Las prioridades así establecidas exigen, como contrapartida, sacrificios en otras áreas, en las que el actual escenario estratégico permite ahorros significativos que no comprometerán la capacidad de la Armada para cumplir su misión.

\section{Un futuro prometedor}

He querido señalar los retos más importantes que tenemos por delante. Son importantes, pero tenemos trazada una línea de actuación que estoy seguro nos garantiza un futuro brillante. La tarea es ilusionante y compensa cualquier esfuerzo y sacrificio, pues de ella depende la futura eficacia de la Armada. Culminarla con éxito exige el esfuerzo constante, coordinado y entusiasta de todos cuantos, hombres o mujeres, de uniforme o de paisano, servimos en esta veterana y, a la vez, joven Institución que es la Armada. Tengo la certeza absoluta de que, en la línea de nuestras mejores tradiciones, la Armada seguirá cumpliendo con eficacia las misiones que tiene encomendadas. 